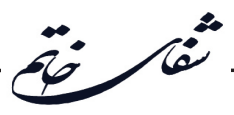

\title{
The Importance of Comprehensive Supportive Cares after Neurosurgical Operations
}

\section{Hadi Khanmoradi $^{*}$, Solmaz Karimi ${ }^{1}$, Soghra Khanmoradi ${ }^{1}$, Ahmadreza Barahimi ${ }^{2}$ Ali Jahanbazi Jahan Abad ${ }^{3}$}

${ }^{1}$ Faculty of Nursing and Midwifery, Zanjan University of Medical Sciences, Zanjan, Iran

${ }^{2}$ Department of Mycology, Faculty of Medical Sciences, Tarbiat Modares University, Tehran, Iran

${ }^{3}$ Shefa Neuroscience Research Center, Khatam Alanbia Hospital, Tehran, Iran

\section{Article Info:}

\section{A BSTRACT}

Introduction: The primary goal of intensive care after neurosurgery is to identify and prevent neurodegeneration by providing supportive activities to keep stable homeostasis. Factors such as surgery, anesthesia, or other illness-related issues influence the postoperative status of patients. In this regard, the nursing and medical staff aware of the neurosurgical procedures should plan special monitoring and follow-up period shortly after the surgery. In many neurosurgical centers, the postoperative care period may be relatively short, like limited craniotomies. However, the occurrence of cerebral edema, intracranial hemorrhage, seizures, or other life-threatening conditions prolongs the postoperative care for several days. Overall, maintaining airway safety, eliminating the need for ventilation, controlling blood circulation and fluid balance, nutrition, sedation control, and analgesics are the mainstays of care. Careful attention to each of these issues is essential to improve neurosurgical outcomes. Conclusion: Robust postoperative care management, particularly in the early stages, is essential to ensure appropriate preoperative conditions. Nurses play a crucial role in monitoring patients following neurosurgical events.

\section{Keywords:}

1. Intensive Care Units

2. Awareness

3. Health

4. Neurosurgery

5. Ventilation

*Corresponding Author: Hadi Khanmoradi

Email: khanmoradi.h@gmail.com 


\section{اهميت مراقبتهاى حمايتى جامع يس از عملهاى جراحى مغز و اعصاب}

هادى خانمر ادى'"، سولماز كريمى'، صغرى خانمرادى'، احمدرضا براهيمى'، على جهانبازى جهان آباد”

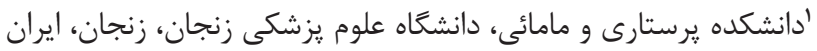

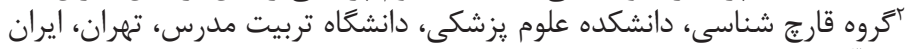

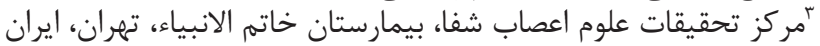

اطلاعات مقاله:

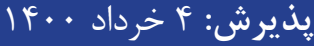

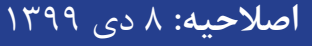

دريافت: له شهريور وqسا

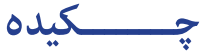

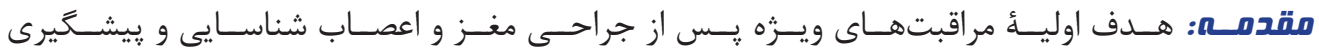

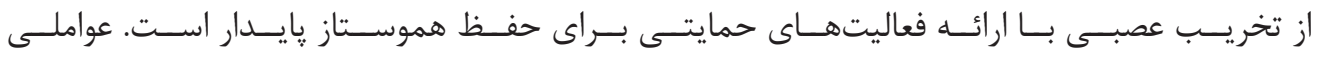

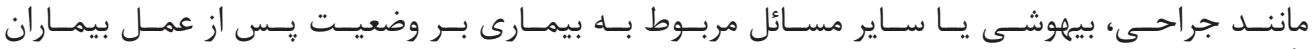

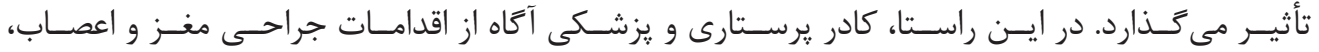

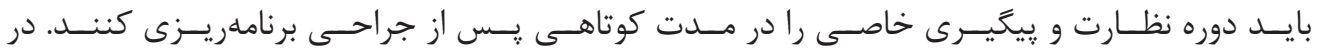

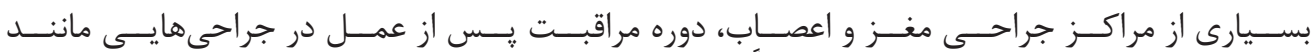

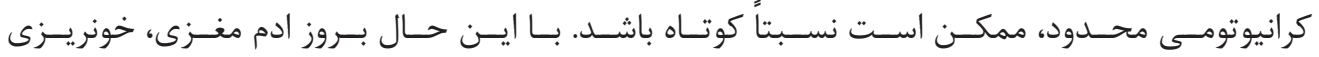

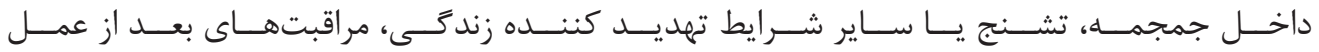

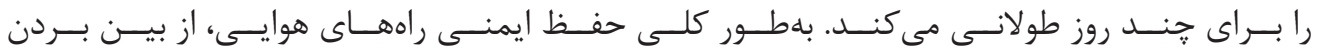

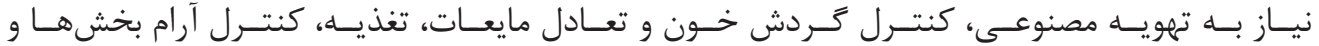

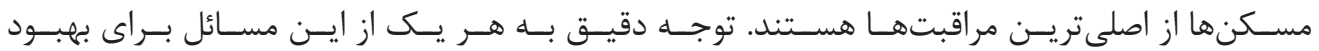

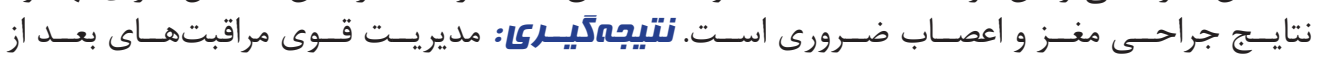

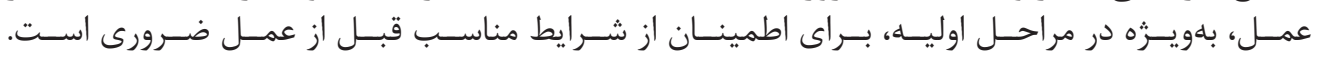

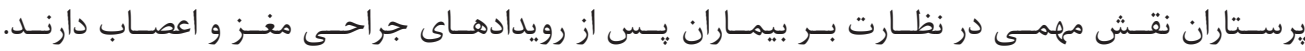

ا - بخشهاى مراقبت ويثه

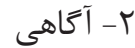

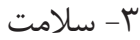
أ

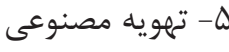




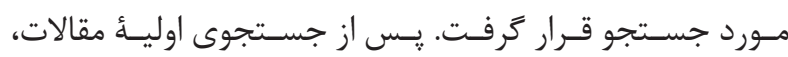

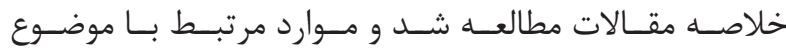

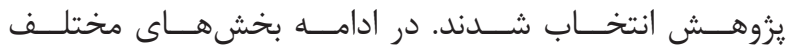

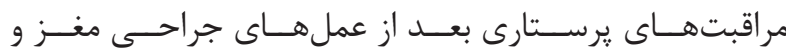

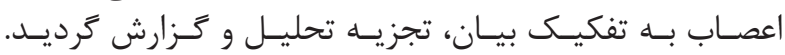

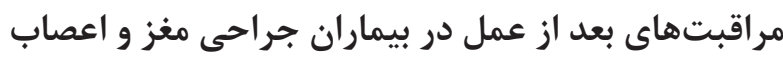

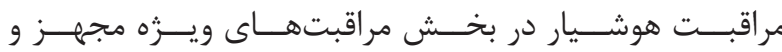

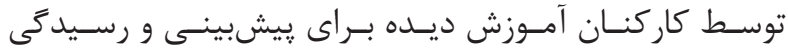

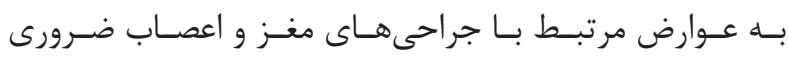

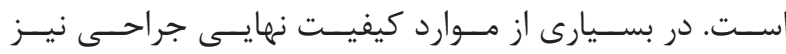

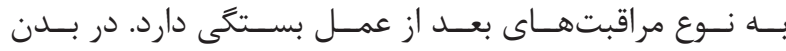

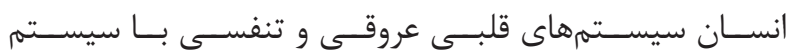

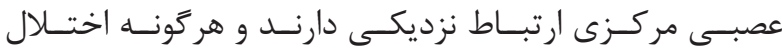

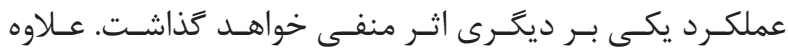

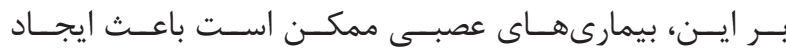

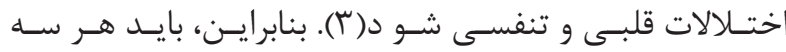

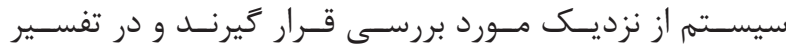

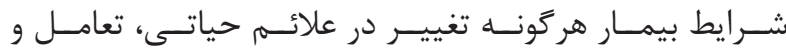

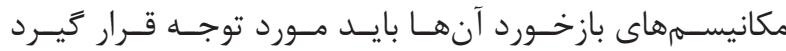

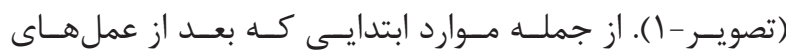

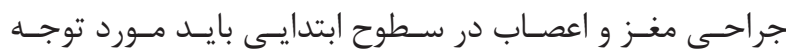

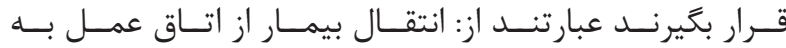

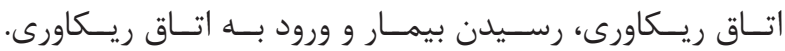

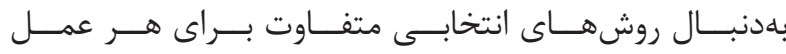

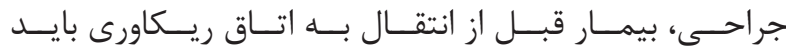

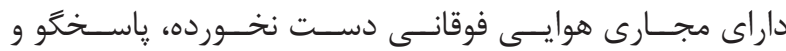

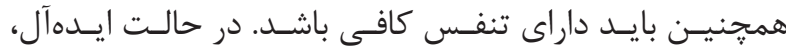

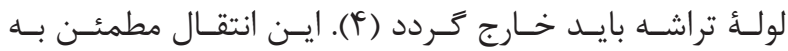

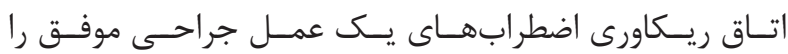

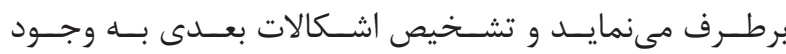

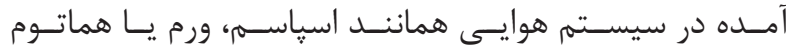

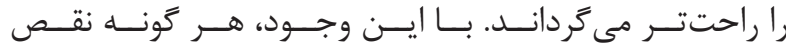

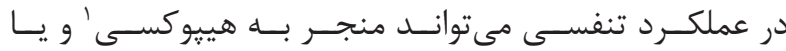

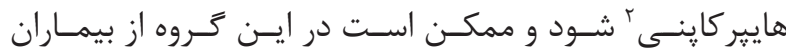

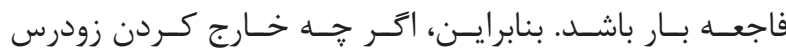

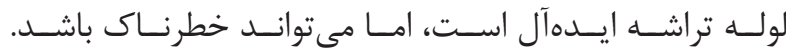

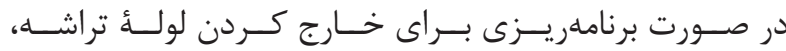

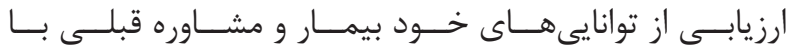

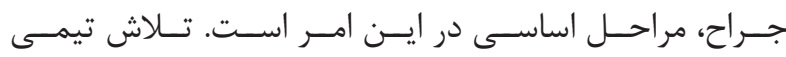

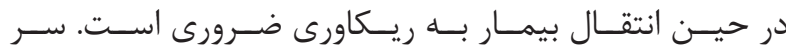

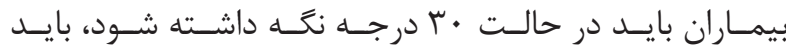

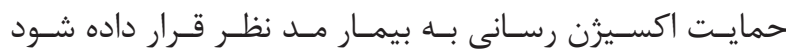

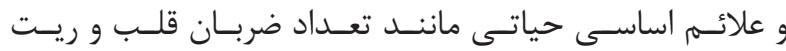

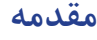

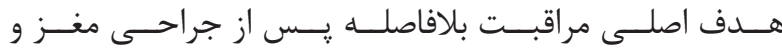

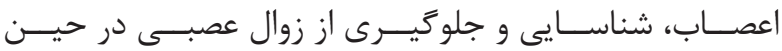

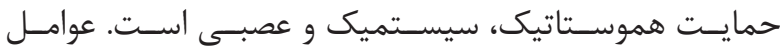

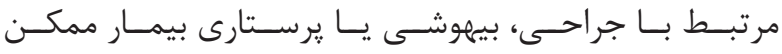

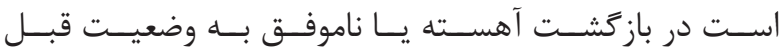

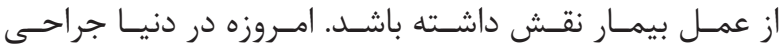

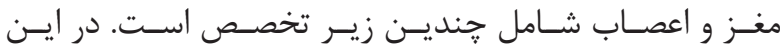

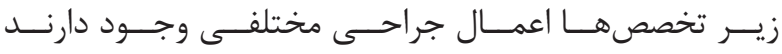

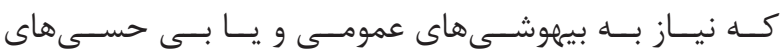

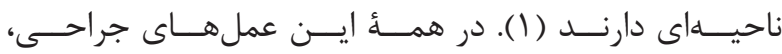

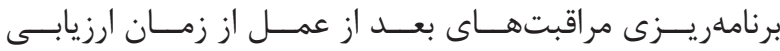

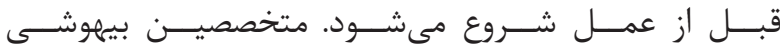

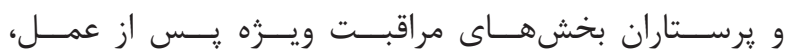

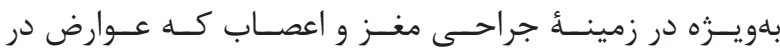

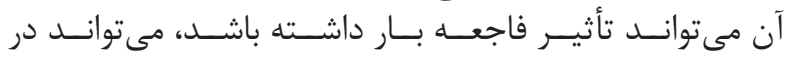

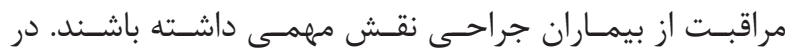

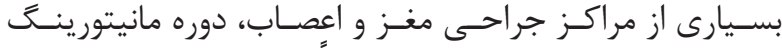

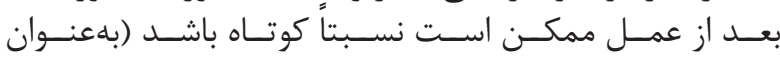

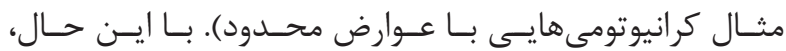

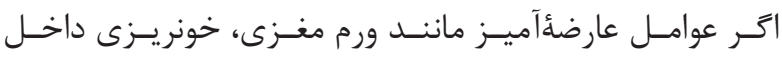

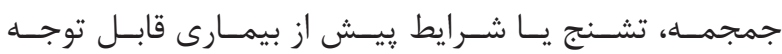

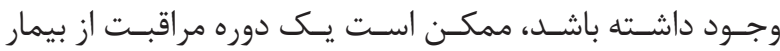

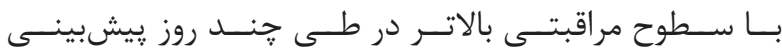

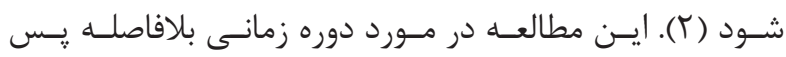

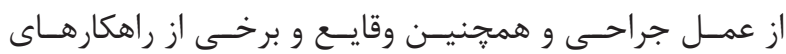

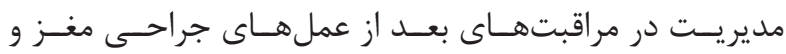

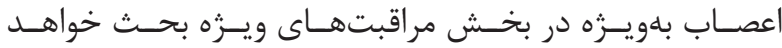

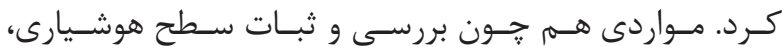

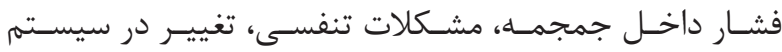

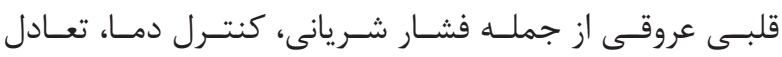

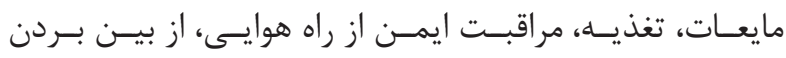

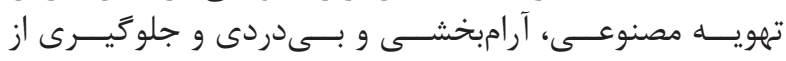

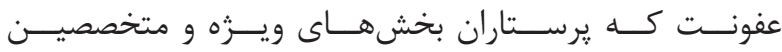

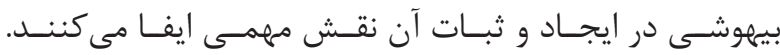

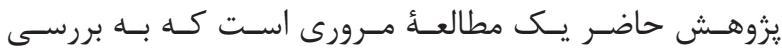

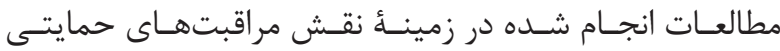

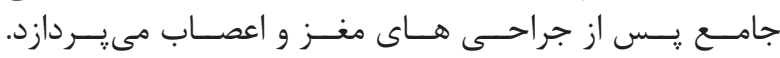

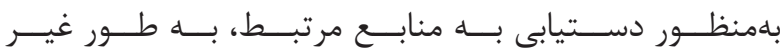

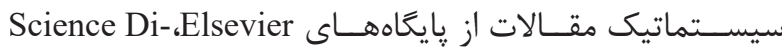

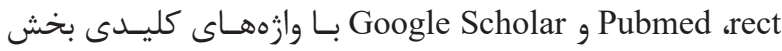

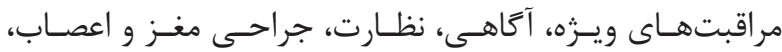

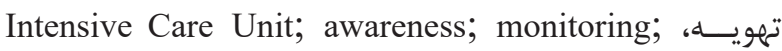

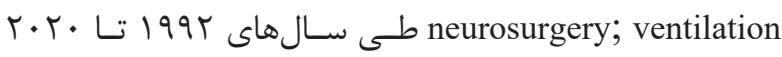




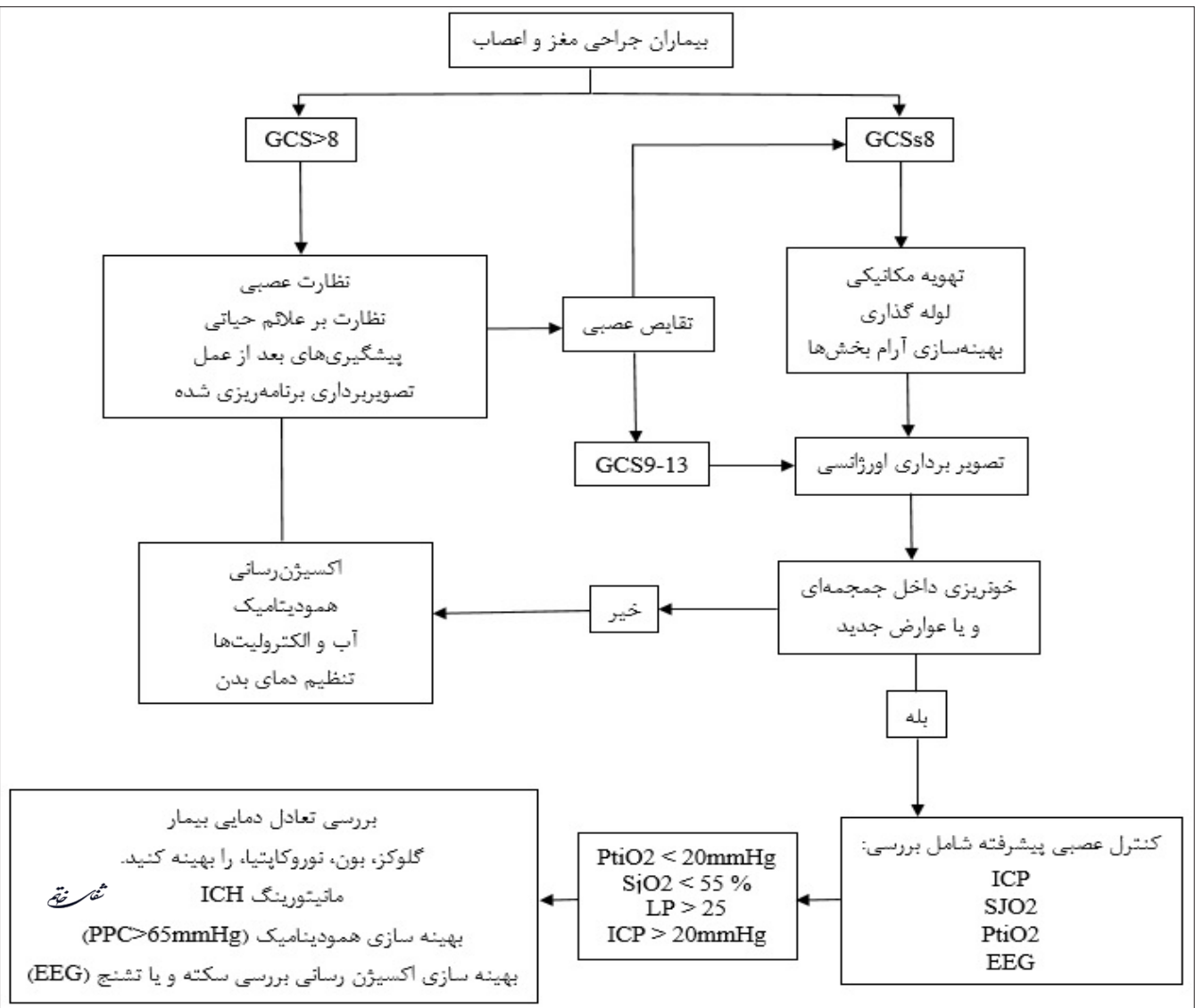

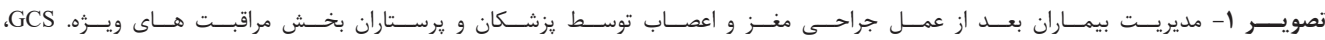

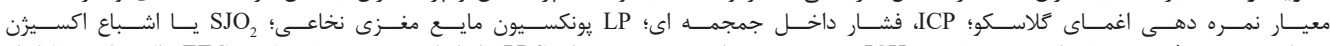

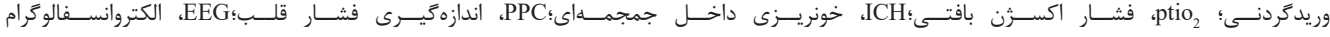

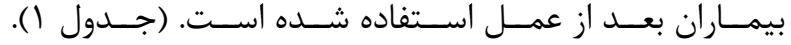

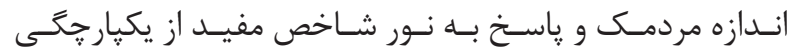

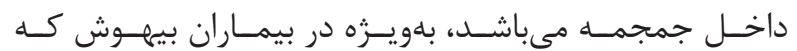

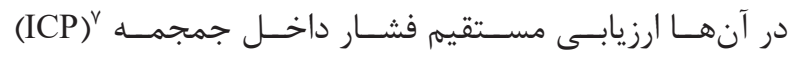

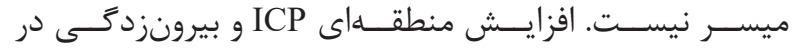

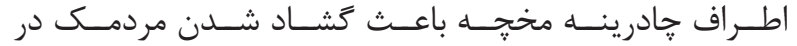

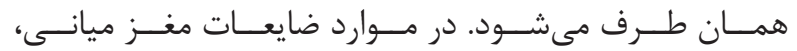

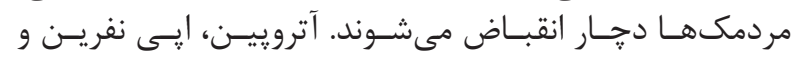

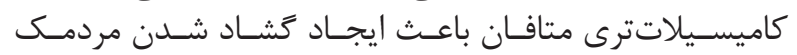

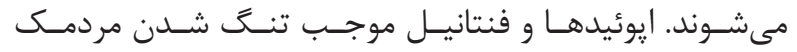

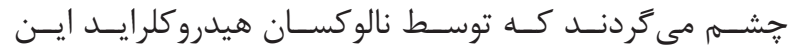

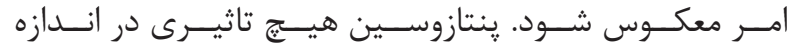

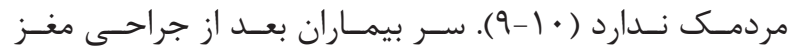

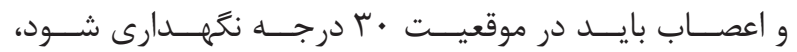

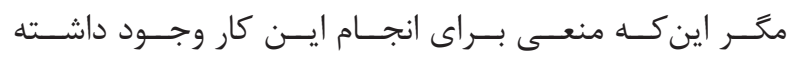

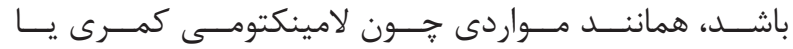

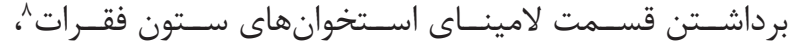

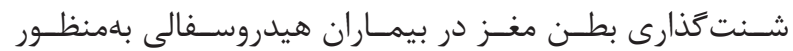

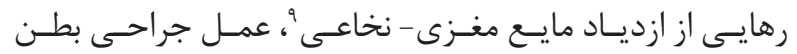

${ }^{3}$ Oscilloscopy

${ }^{4}$ Capnograph

${ }^{5}$ Roentgenogram

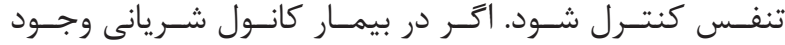

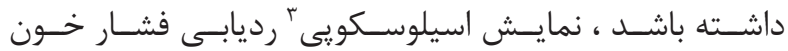

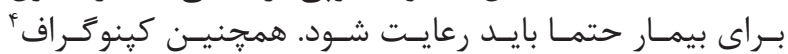

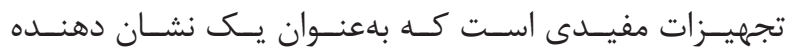

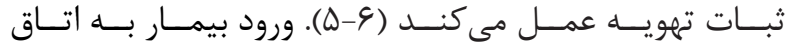

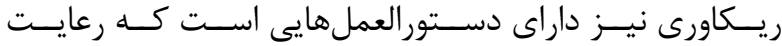

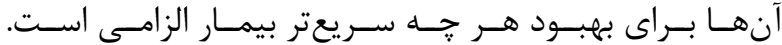

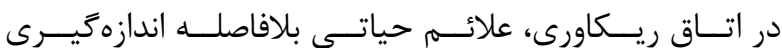

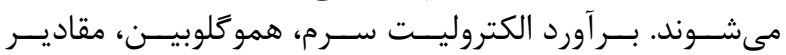

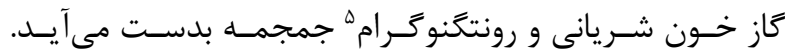

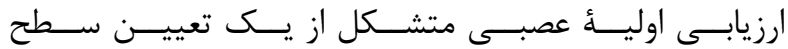

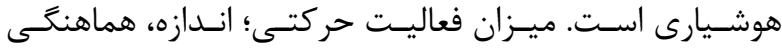

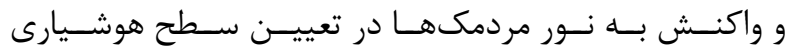

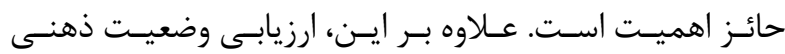

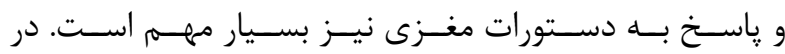

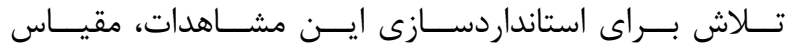

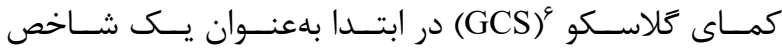

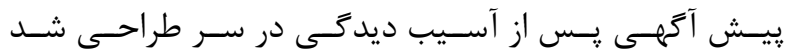

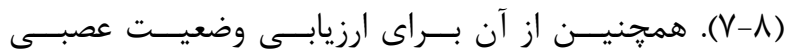

\footnotetext{
${ }^{6}$ Glasgow Coma Scale

${ }^{7}$ Intracranial pressure

${ }^{8}$ lumbar laminotomy

${ }^{9}$ Ventricular shunting
} 


\begin{tabular}{|c|c|c|}
\hline معيار & وضعيت & 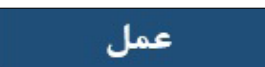 \\
\hline r & بيمار حشمهايش راخود به خود باز كند & \multirow{4}{*}{ باز كردن جشمها } \\
\hline r & بيمار در صاسيخ به صدا حشمهايش را باز كند & \\
\hline r & بيمار در صاسخ به درد حشمهايش را باز كند & \\
\hline 1 & بيمار اصلا حشمهايش را باز نكند & \\
\hline$\Delta$ & بيمار كاملا به زمان و مكان و اشخاص اطر اف آكاهى دارد & \multirow{5}{*}{ 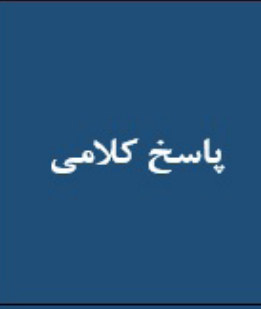 } \\
\hline f & بيمار كيج است & \\
\hline r & بيمار كلمات نامربوط به زبان مىآورد & \\
\hline r & بيمار كلمات نامفهوم به زبان مىآورد & \\
\hline 1 & بيمار صحبت نمىكند & \\
\hline$\varepsilon$ & بيمار دستورات را اجراء مىكند & \multirow{6}{*}{ اسخ حركتى } \\
\hline$\Delta$ & بيمار محل درد را تشخيص مىدهد & \\
\hline f & بيمار خود را ازز محرك دردناك دور مىكند & \\
\hline r & بيمار در صاسخ به محر كى درناك، اندامهايش را به وضعيت فلكسيون(خم شدن) در مىآورد & \\
\hline r & بيمار در هاسخ به محرك دردناك، اندامهايش را به وضعيت اكستنشن(باز شدن) در مىآورد & \\
\hline 1 & 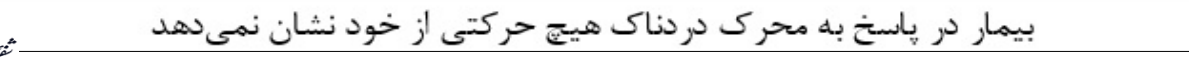 & \\
\hline
\end{tabular}

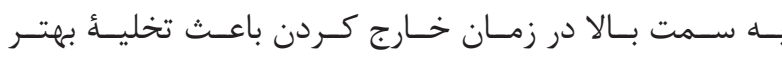

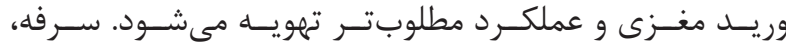

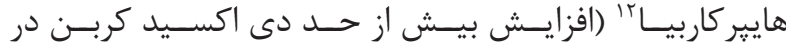

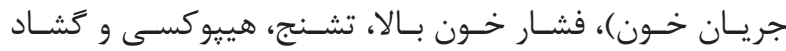

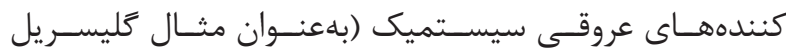

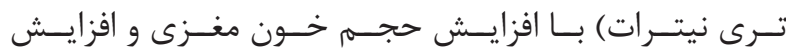

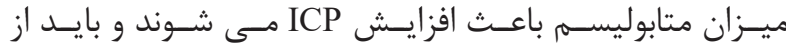

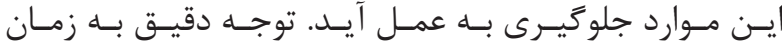

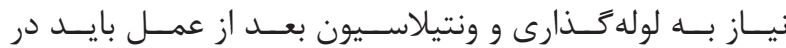

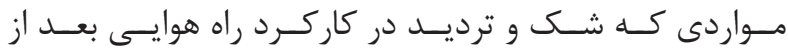

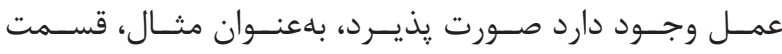

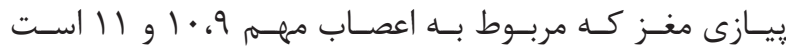

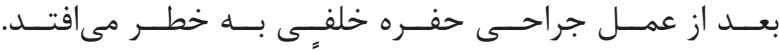

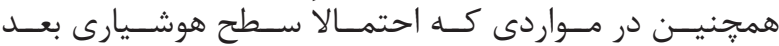

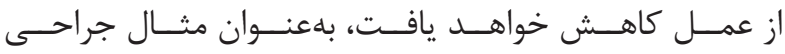

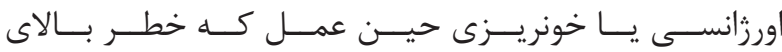

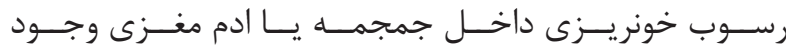

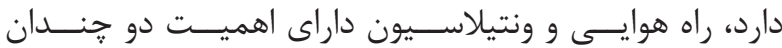

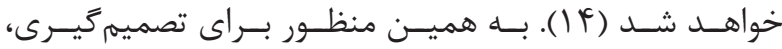

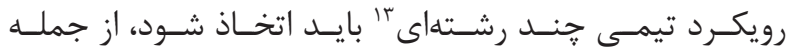

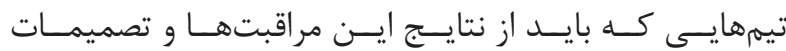

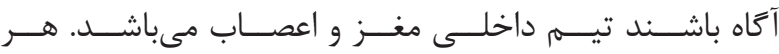

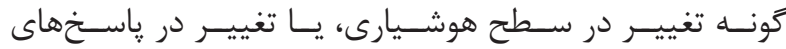

${ }^{10}$ Carotid endarterectomy

${ }^{11}$ Remifentanil

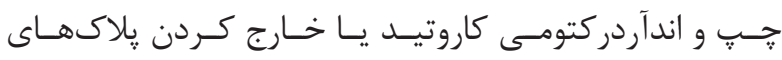

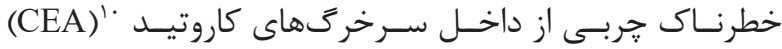

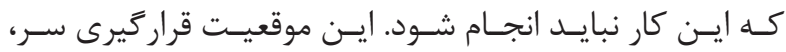

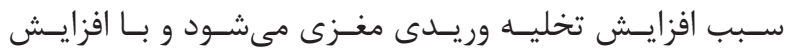

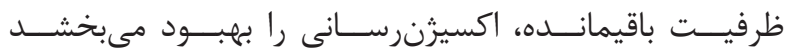

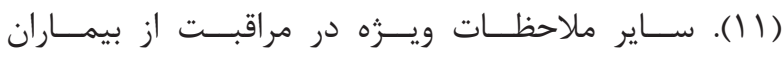

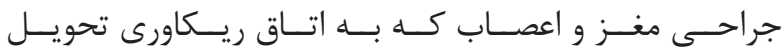

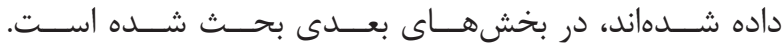

تصميمَيرى در مورد زمان خارج كردن لولئ تراشه

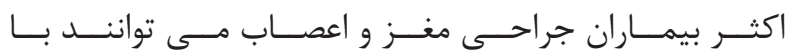

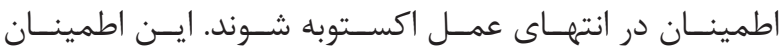

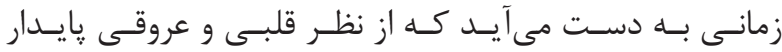

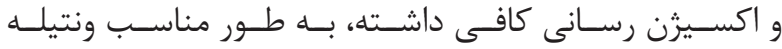

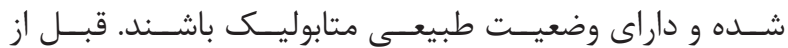

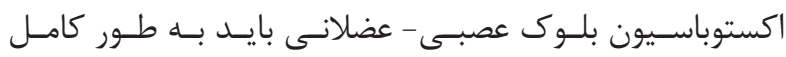

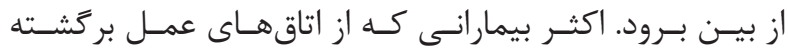

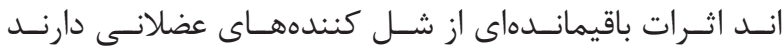

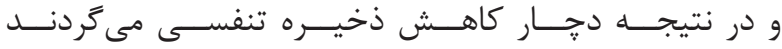

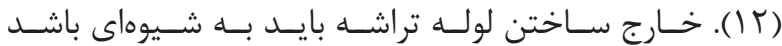

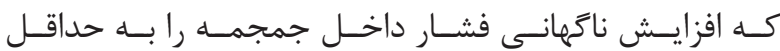

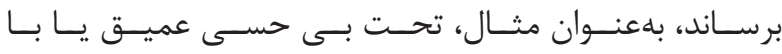

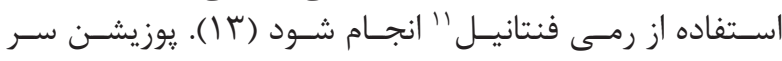

${ }^{12}$ Hypercarbia

${ }^{13}$ Multi-disciplinary team 


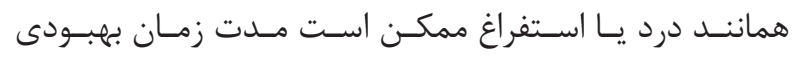

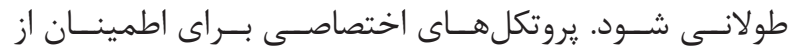

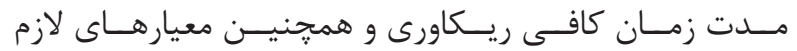

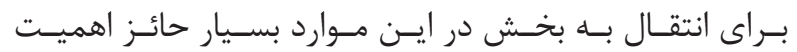

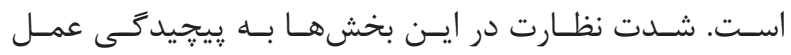

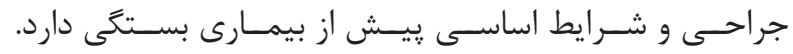

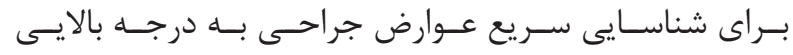

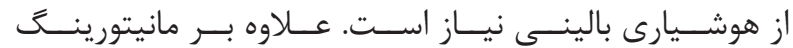

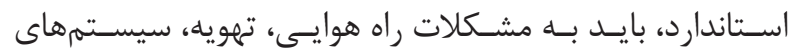

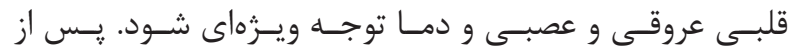

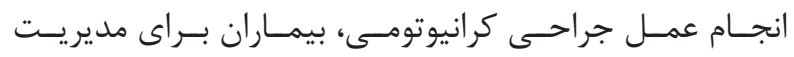

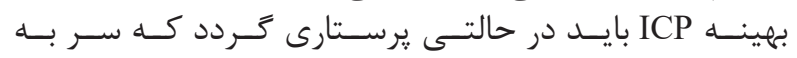

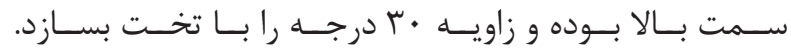

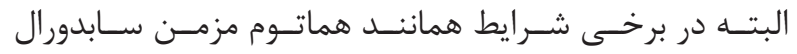

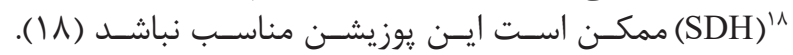

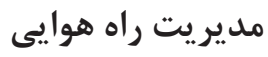

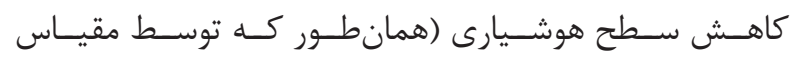

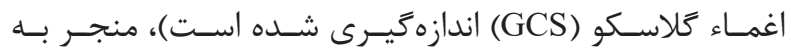

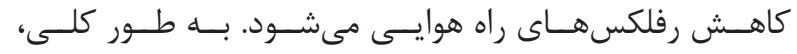

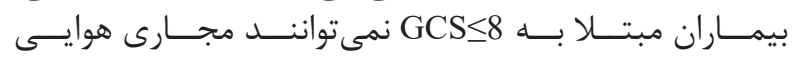

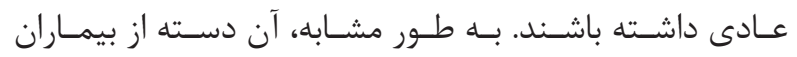

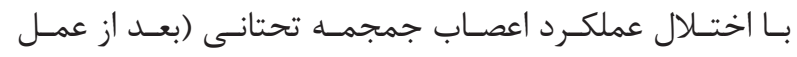

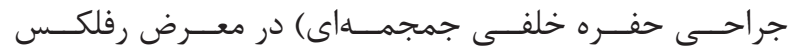

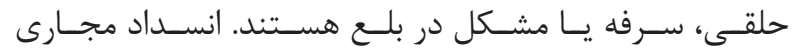

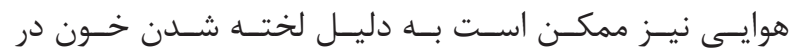

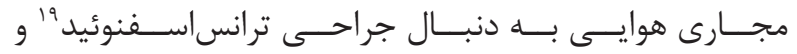

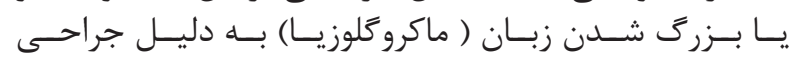

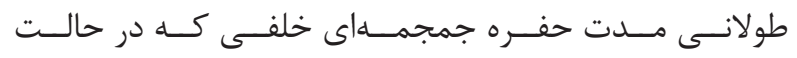

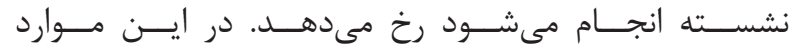

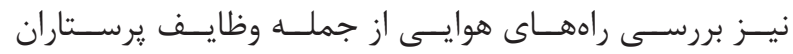

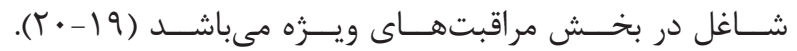

تهويه

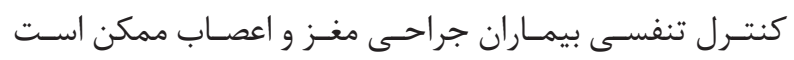

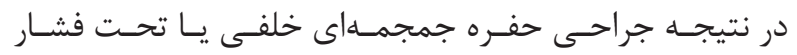

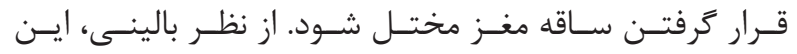

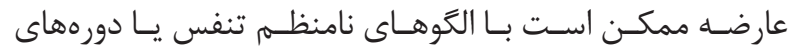

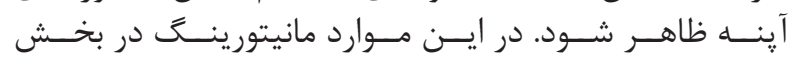

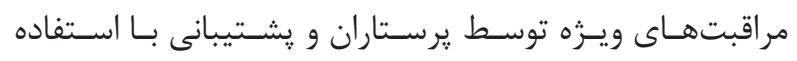

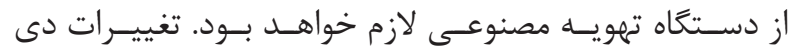

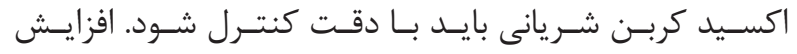

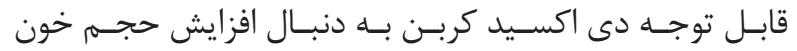

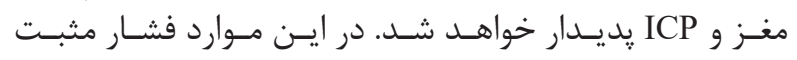

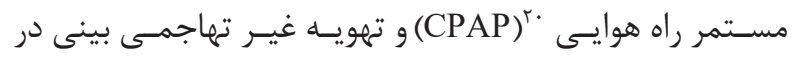

${ }^{14}$ Royal College of Anaesthetists

${ }^{15}$ Post Anaesthetic Care Unit

${ }^{16}$ Sepsis

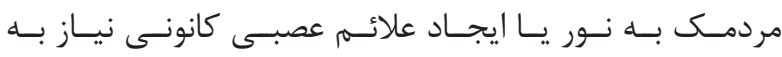

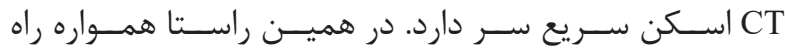

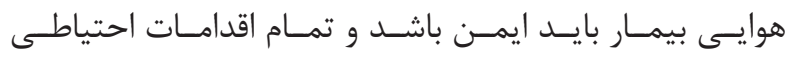

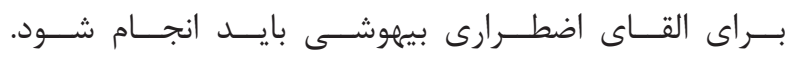

\section{نظارت بعد از عمل و انتخاب سطح مراقبت}

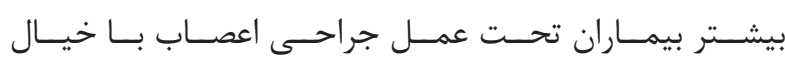

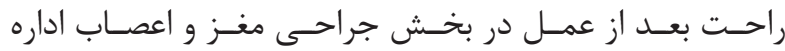

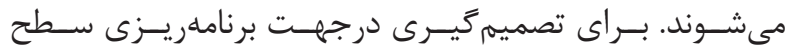

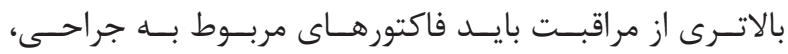

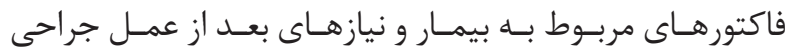

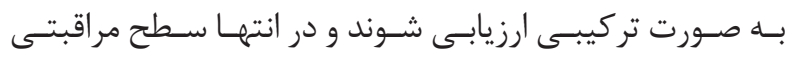

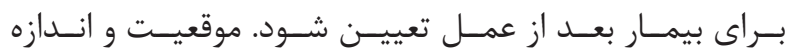

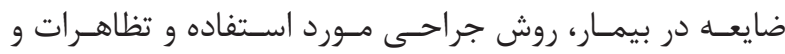

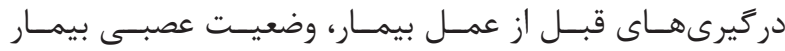

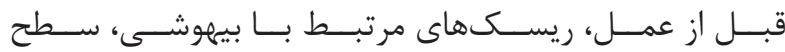

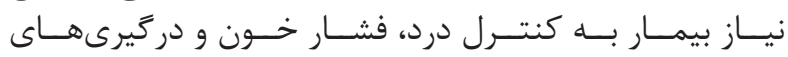

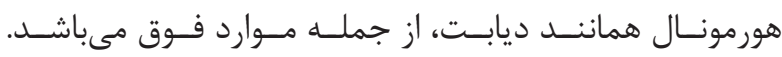

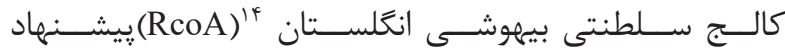

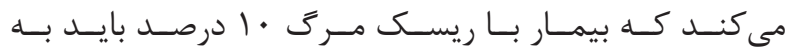

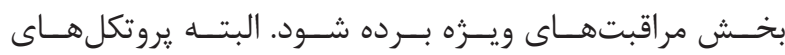

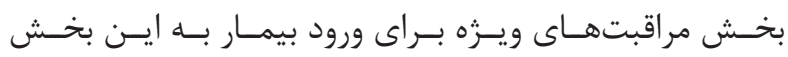

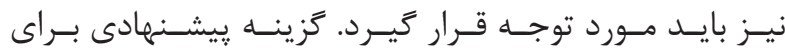

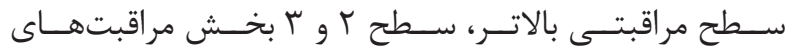

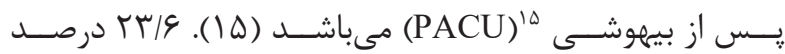

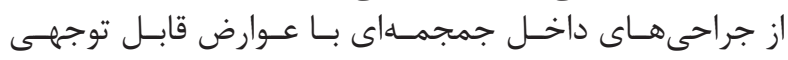

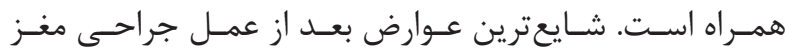

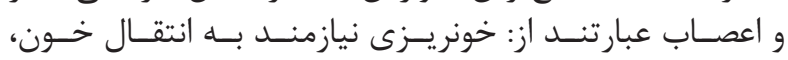

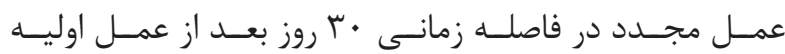

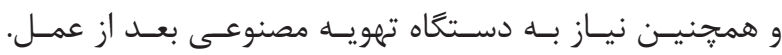

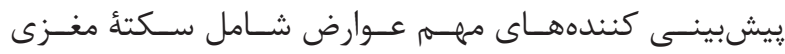

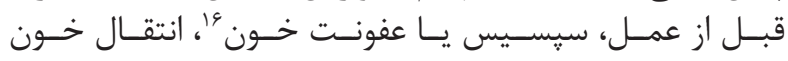

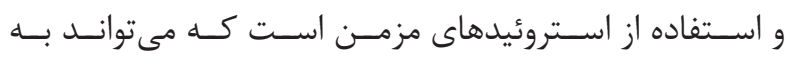

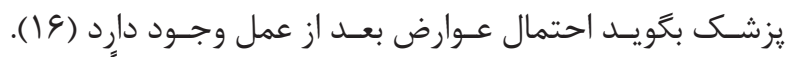

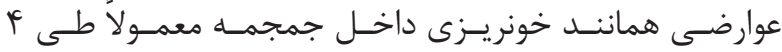

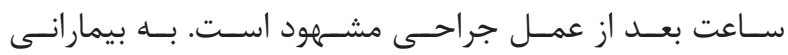

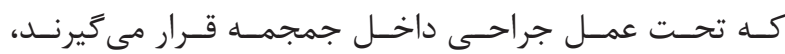

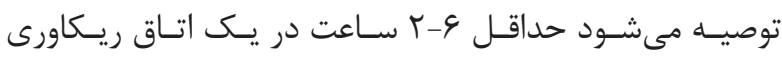

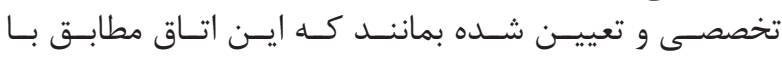

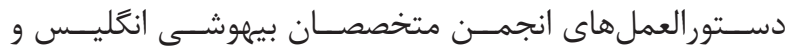

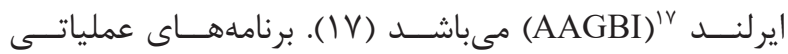

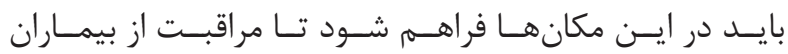

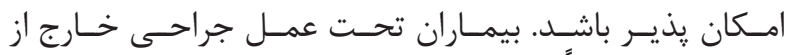

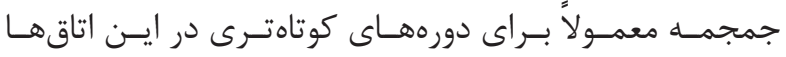

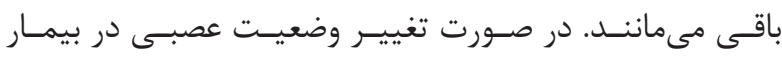

\footnotetext{
${ }^{17}$ Association of Anaesthetists of Great Britain and Ireland

${ }^{18}$ Chronic subdural hematoma

${ }^{19}$ Transsphenoidal surgery

${ }^{20}$ Continuous positive airway pressure
} 


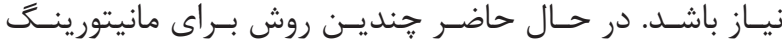

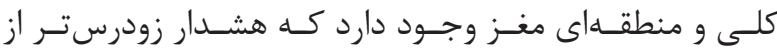

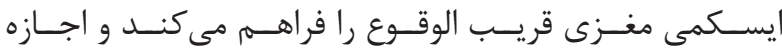

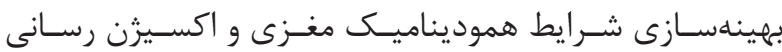

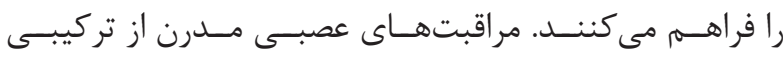

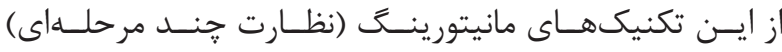

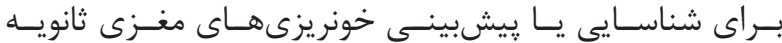

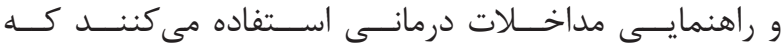

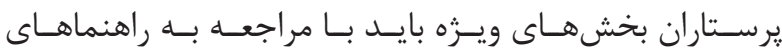

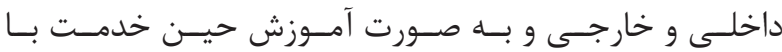

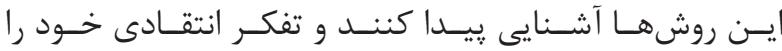

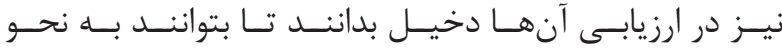

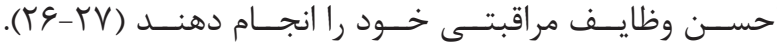

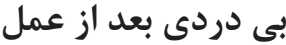

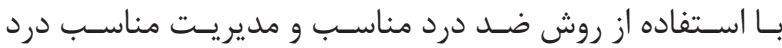

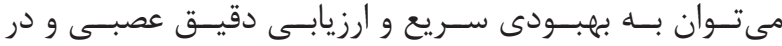

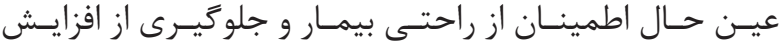

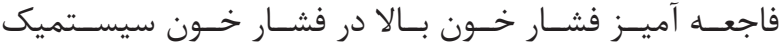

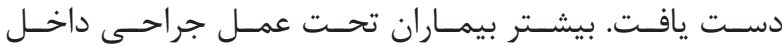

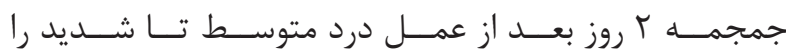

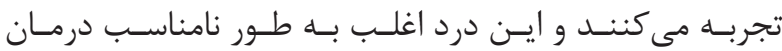

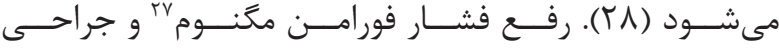

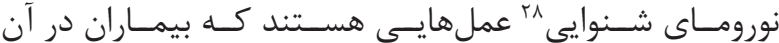

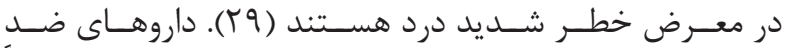

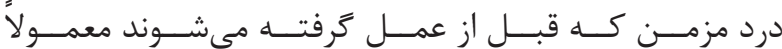

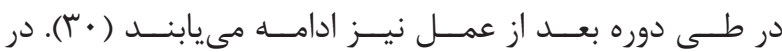

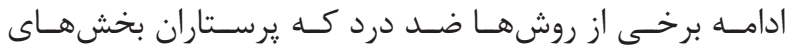

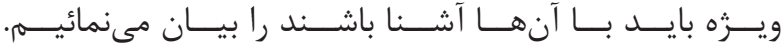

ايوئيدها

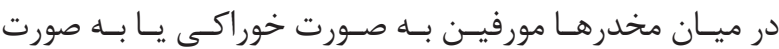

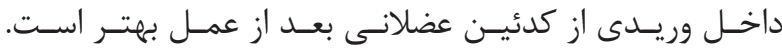

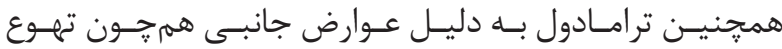

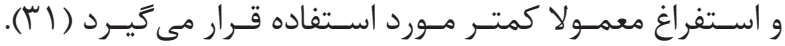

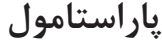

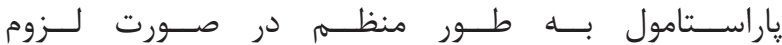

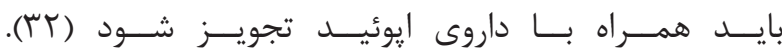

\section{داروهاى ضدالتهابى غير استروئيدى}

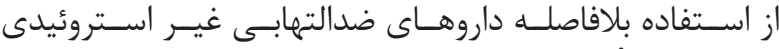

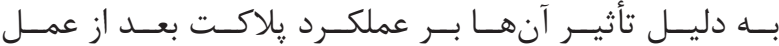

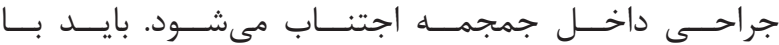

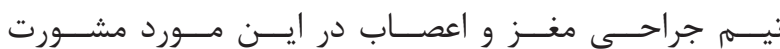

\footnotetext{
${ }^{21}$ Intracranial hemorrhage

${ }^{22}$ Brain perfusion

${ }^{23}$ Vasopressors

${ }^{24}$ Obstructive Hydrocephalus
}

بيمـاران بـا كاهـش ظرفيـت تهويـهـ تنفسـى لازم اسـت ( ( آ).

\section{سيستم قلبى عروقى}

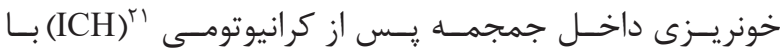

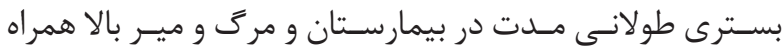

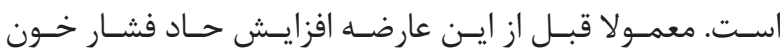

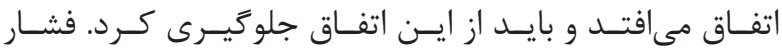

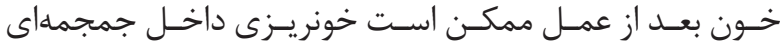

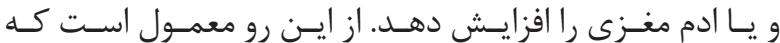

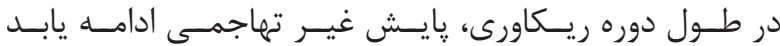

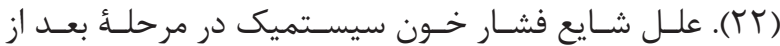

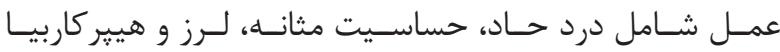

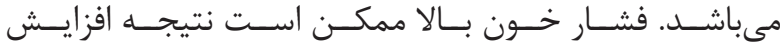

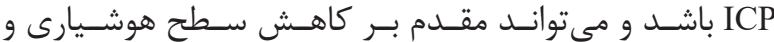

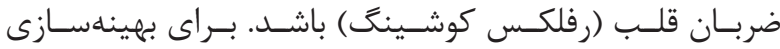

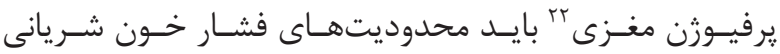

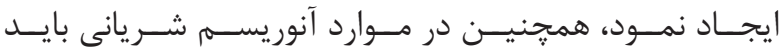

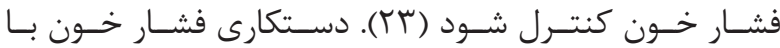

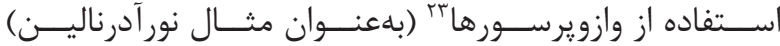

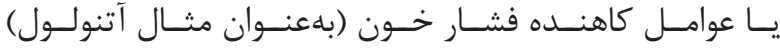

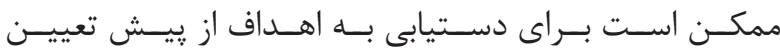

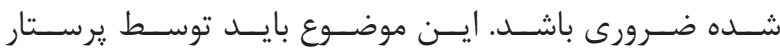

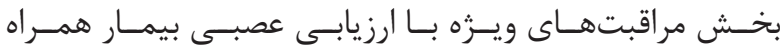

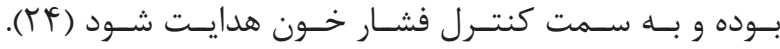

سيستم عصبسى

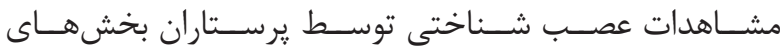

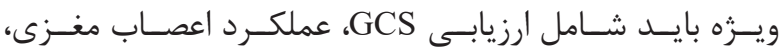

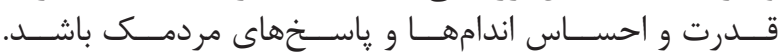

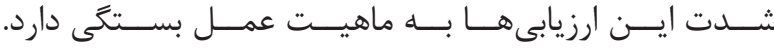

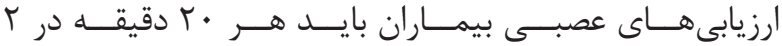

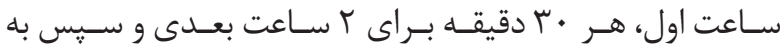

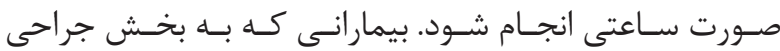

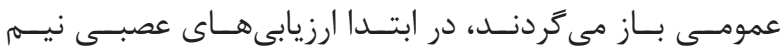

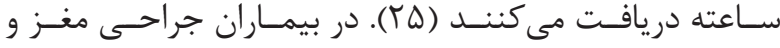

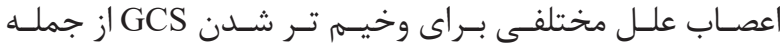

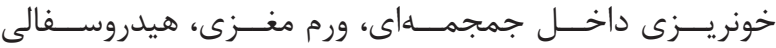

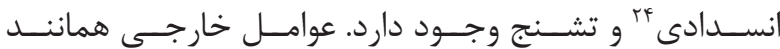

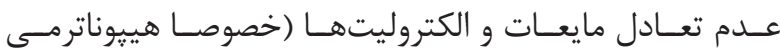

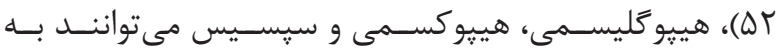

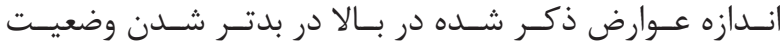

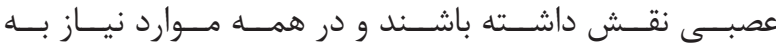

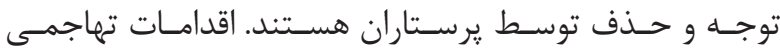

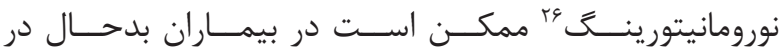

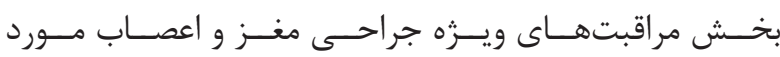

${ }^{25}$ Hyponatremia

${ }^{26}$ Neuromonitoring

${ }^{27}$ Foreman Magnum

${ }^{28}$ Acoustic Neuroma Surgery 


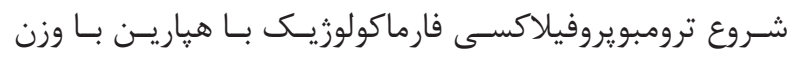

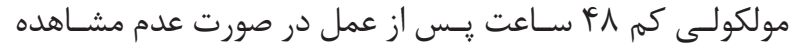

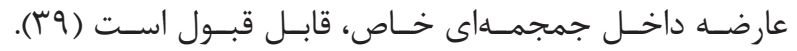

\section{آنتىبيوتيكها}

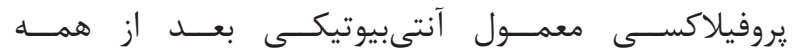

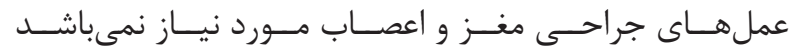

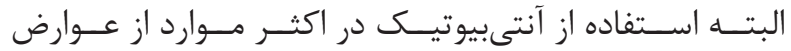

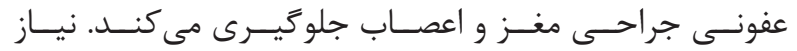

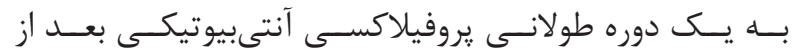

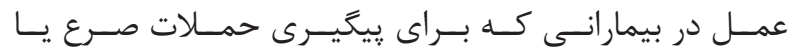

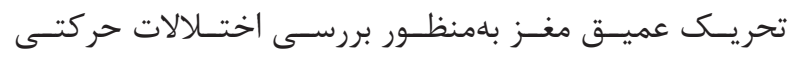

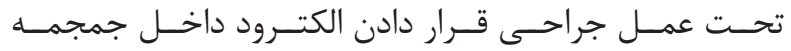

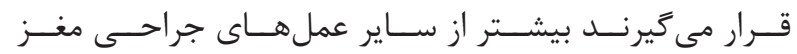

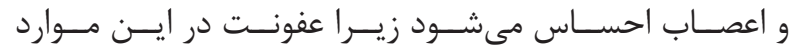

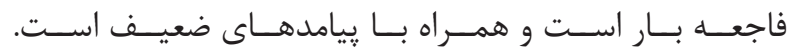

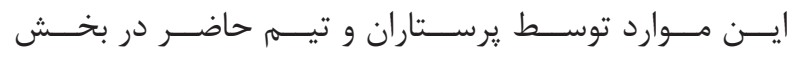

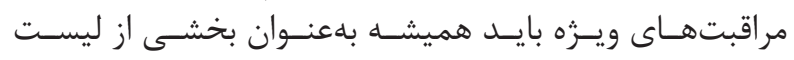

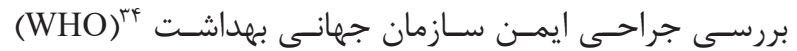

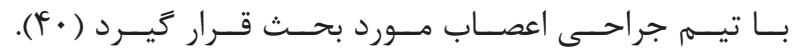

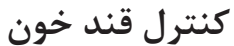

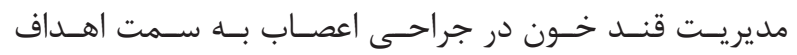

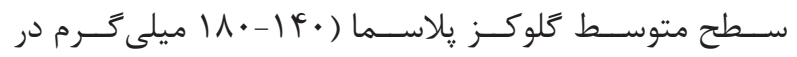

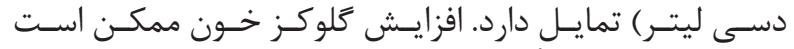

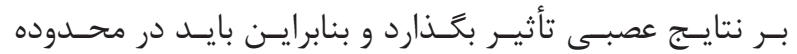

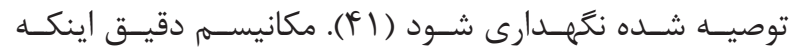

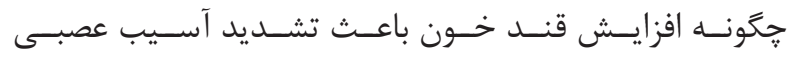

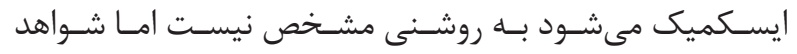

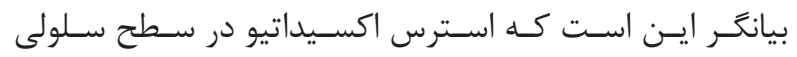

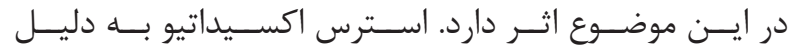

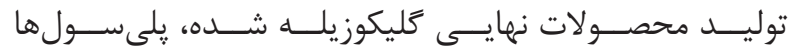

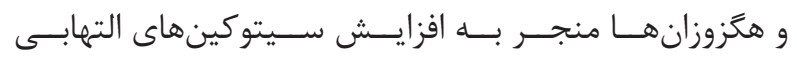

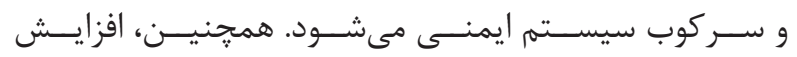

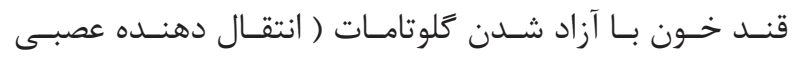

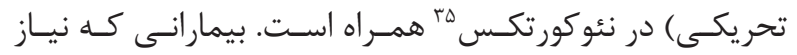

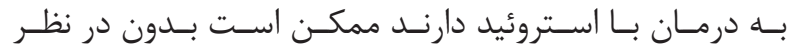

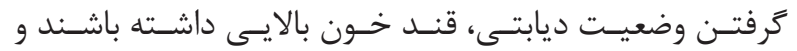

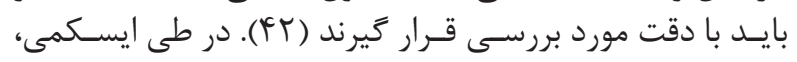

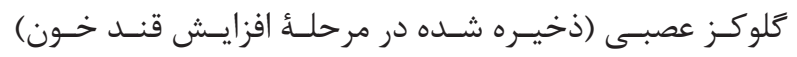

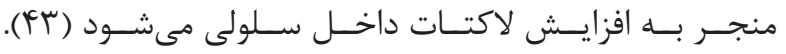

\section{جايكز ينى هور مون}

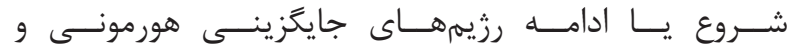

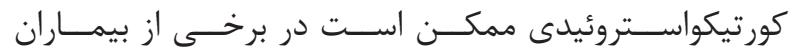

\footnotetext{
${ }^{29}$ Postoperative nausea and vomiting

${ }^{30}$ Infratentorial surgery

${ }^{31}$ Venous thromboembolism

${ }^{32}$ Intermittent pneumatic compression
}

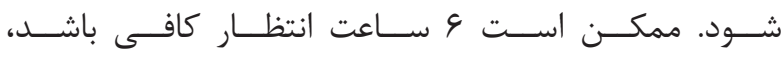

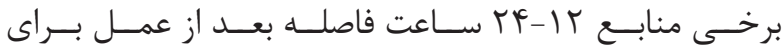

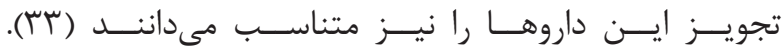

بى حسى موضعى

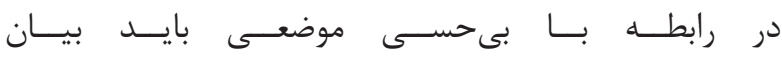

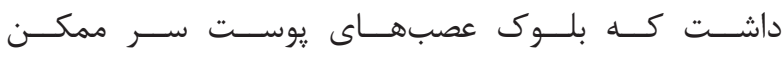

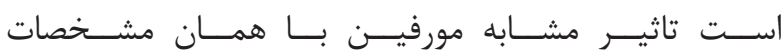

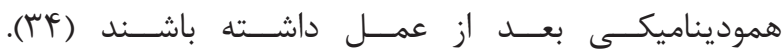

ديغر مكملها

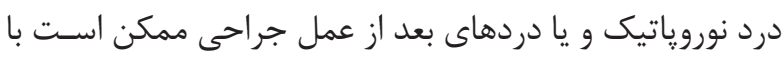

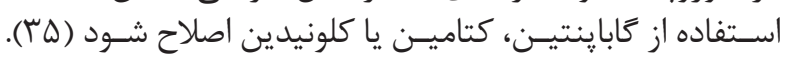

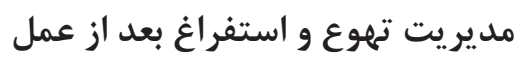

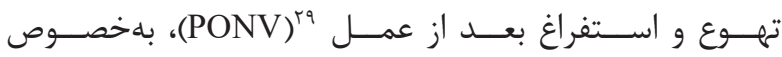

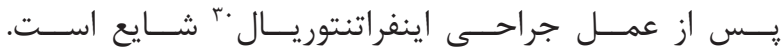

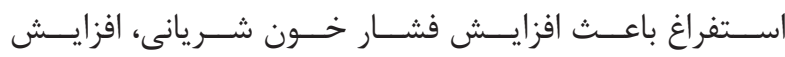

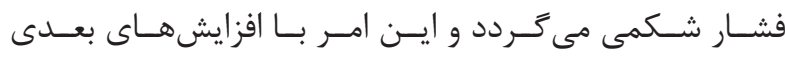

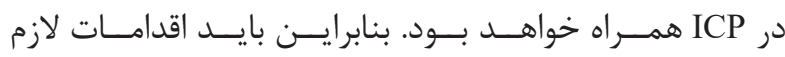

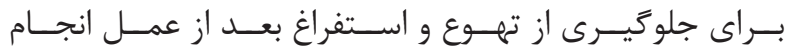

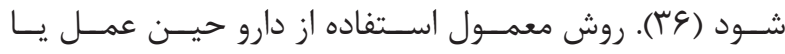

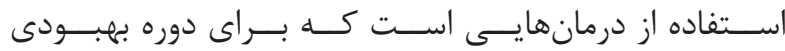

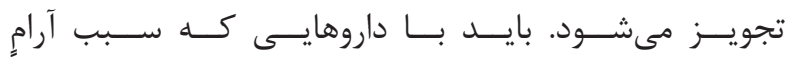

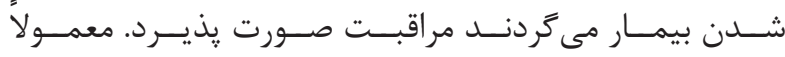

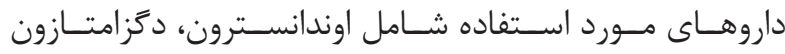

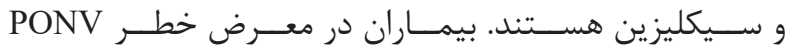

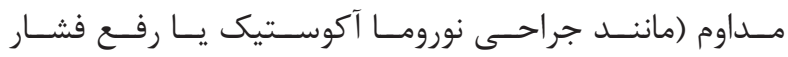

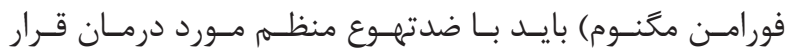

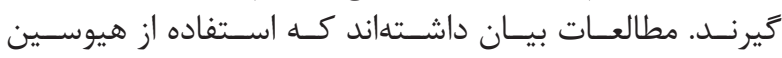

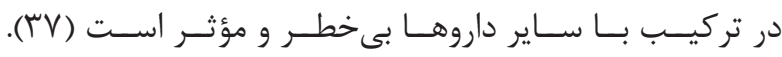

\section{ييشگيرى از ترومبو آمبولى وريدى}

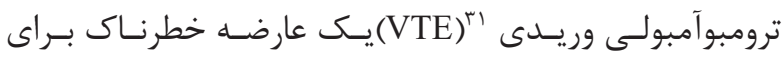

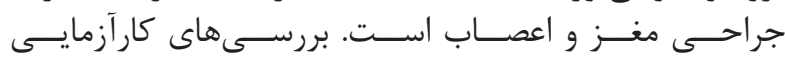

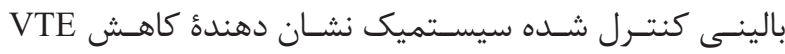

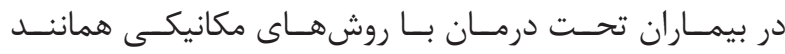

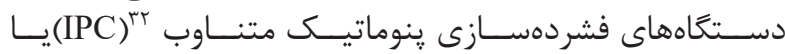

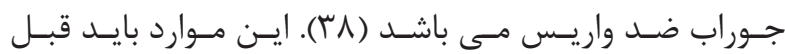

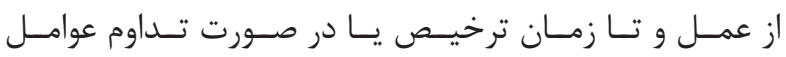

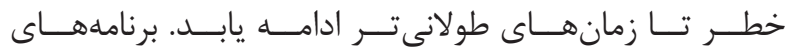

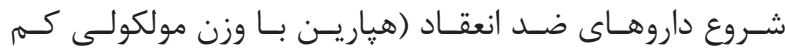

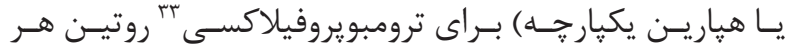

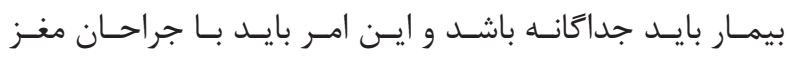

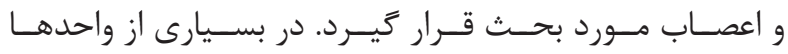

\footnotetext{
${ }^{33}$ Thromboprophylaxis

${ }^{34}$ World Health Organization

${ }^{35}$ Neocortex
} 


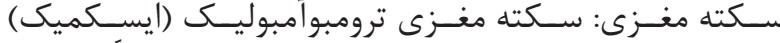

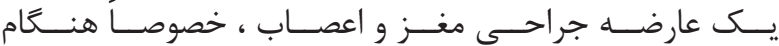

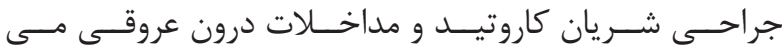

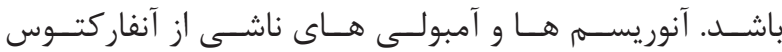

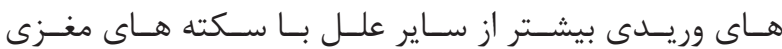

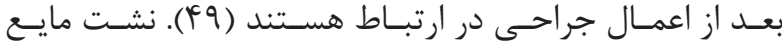

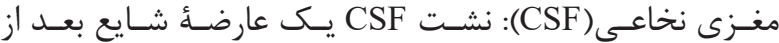

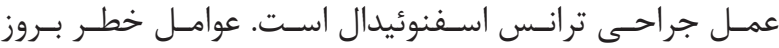

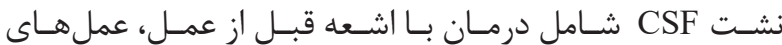

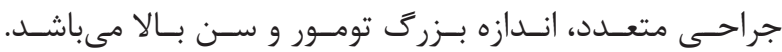

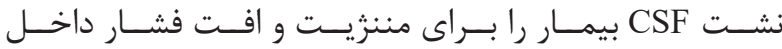

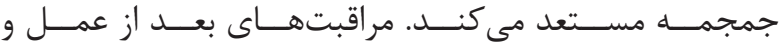

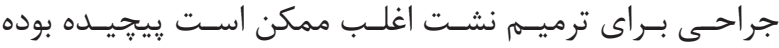

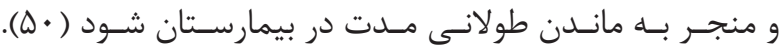

\section{عدم تعادل مايعات و الكتروليتها يس از عمل}

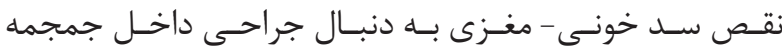

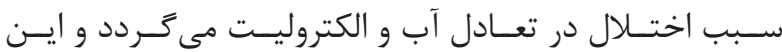

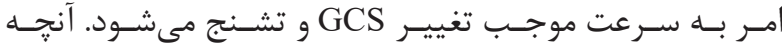

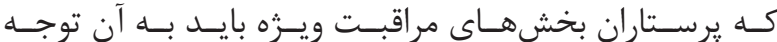

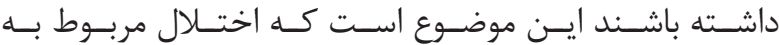

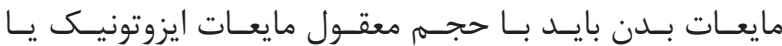

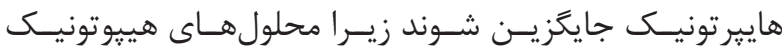

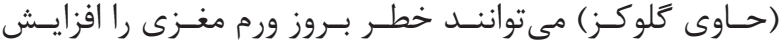

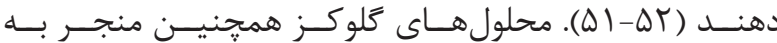

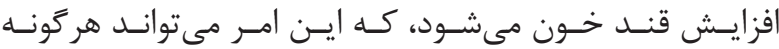

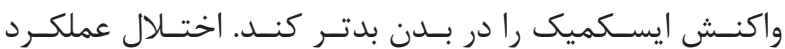

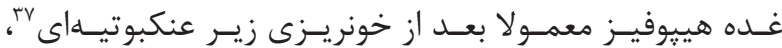

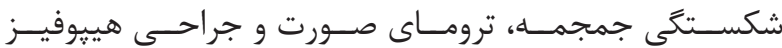

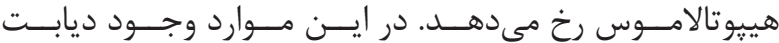

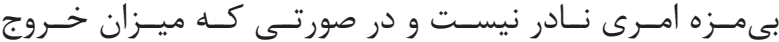

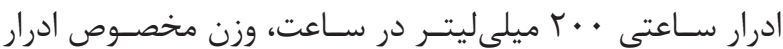

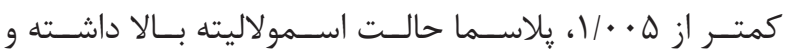

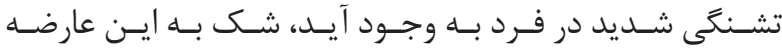

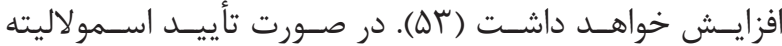

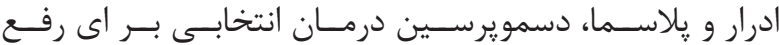

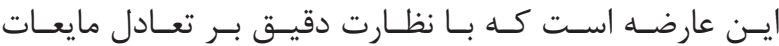

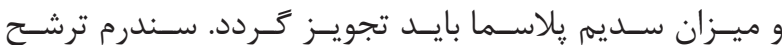

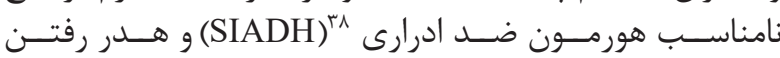

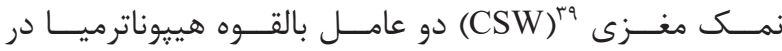

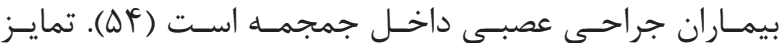

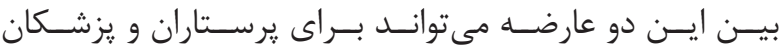

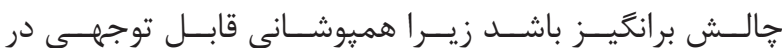

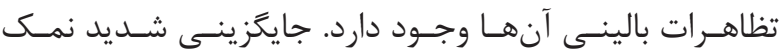

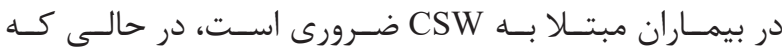

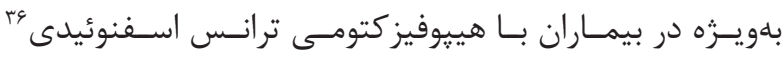

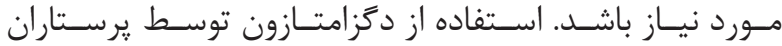

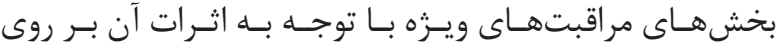

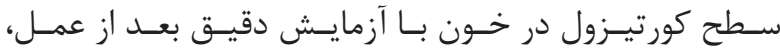

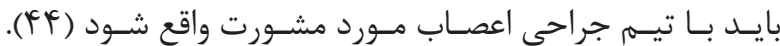

\section{تغذيه}

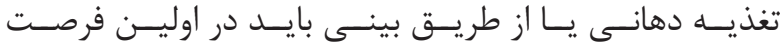

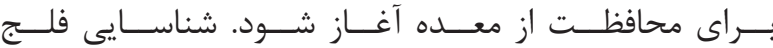

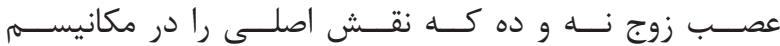

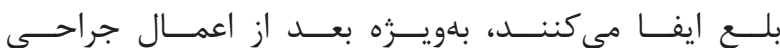

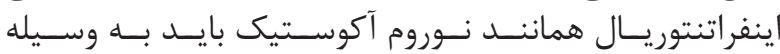

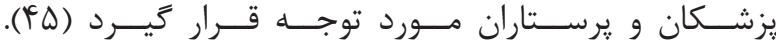

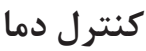

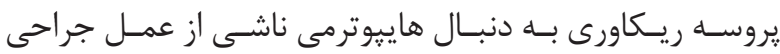

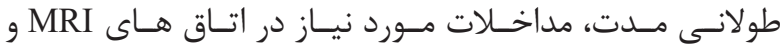

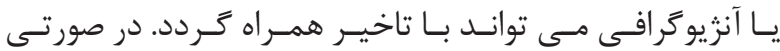

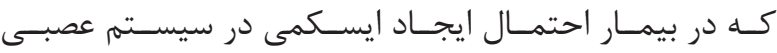

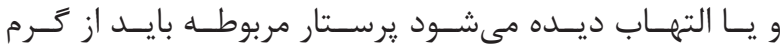

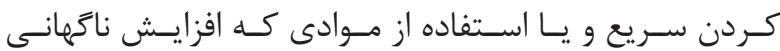

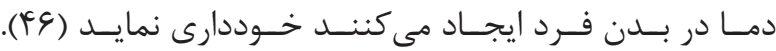

\section{عوارض شايع بعد از عمل جراحى مغز و اعصاب كاهش سطح هوشيارى}

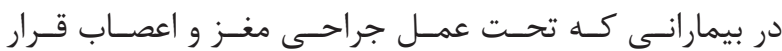

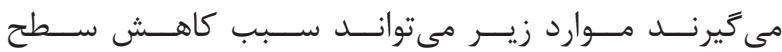

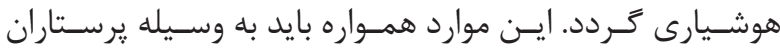

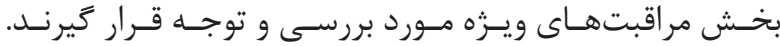

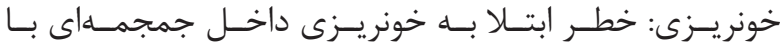

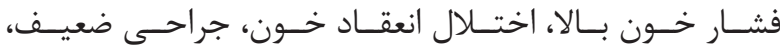

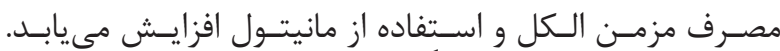

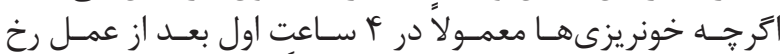

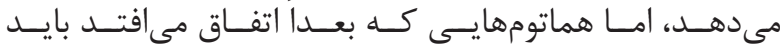

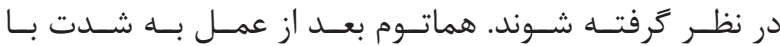

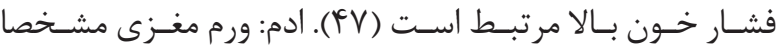

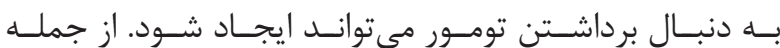

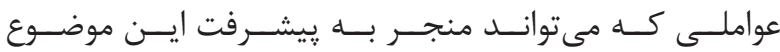

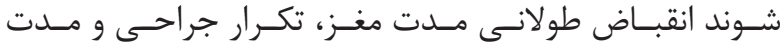

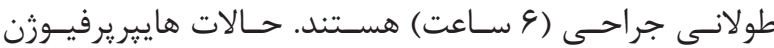

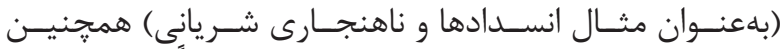

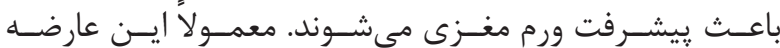

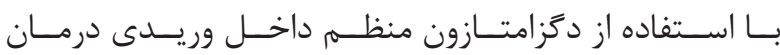

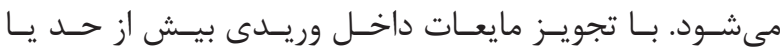

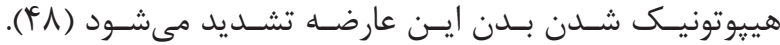

${ }^{38}$ Syndrome of inappropriate antidiuretic hormone secretion

${ }^{39}$ Cerebral Salt-Wasting Syndrome 


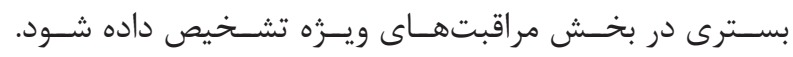

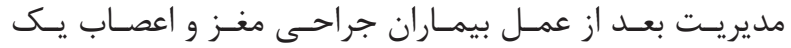

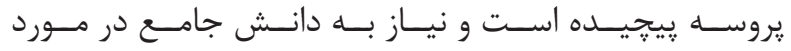

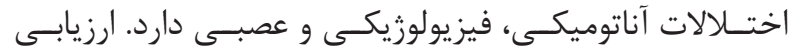

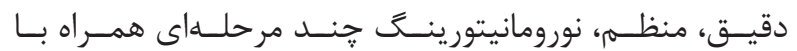

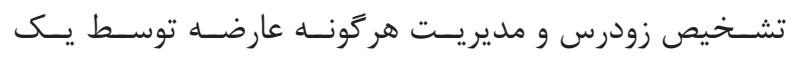

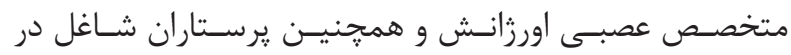

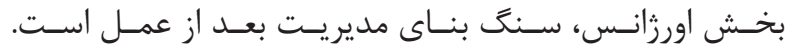

1. Tagaytayan R, Kelemen A, Sik-Lanyi C. Augmented reality in neurosurgery. Archives of medical science: AMS. 2018 Apr; 14(3): 572.

2. Cassir N, De La Rosa S, Melot A, Touta A, Troude L, Loundou A. Risk factors for surgical site infections after neurosurgery: A focus on the postoperative period. American journal of infection control. 2015 Dec 1; 43(12): 1288-91.

3. Tobaldini E, Colombo G, Porta A, Montano N. The Autonomic Nervous System. InStress Challenges and Immunity in Space 2020 (pp. 123-143). Springer, Cham.

4. Smith W, Finley A, Ramsay J. Postoperative Respiratory Failure and Treatment. InPrinciples and Practice of Anesthesia for Thoracic Surgery 2019 (pp. 895-923). Springer, Cham.

5. Reynolds J. The nurse-patient relationship in the postanaesthetic care unit. Nursing Standard. 2009 Dec 16;24.

6. McKinnon M, Donnelly F, Perry J. Experiences of Post Anaesthetic Recovery Nurses facilitating Advanced Directives in the immediate post anaesthetic period: A phenomenological study. Journal of Advanced Nursing. 2020 Mar 18.

7. Awad IT, Chung F. Factors affecting recovery and discharge following ambulatory surgery. Canadian Journal of Anesthesia. 2006 Sep 1; 53(9): 858-72.

8. Zelcer J, Wells DG. Anaesthetic-related recovery room complications. Anaesthesia and intensive care. 1987 May; 15(2): 168-74.

9. Ortega-Perez S, Shoyombo I, Aiyagari V, Atem F, Hill M. Pupillary light reflex variability as a predictor of clinical outcomes in subarachnoid hemorrhage. Journal of Neuroscience Nursing. 2019 Aug 1; 51(4): 171-5.

10. Romagnosi F, Bongiovanni F, Oddo M. Eyeing up the injured brain: automated pupillometry and optic nerve sheath diameter. Current opinion in critical care. 2020 Apr 1; 26(2): 115-21.

11. Ng I, Lim J, Wong HB. Effects of head posture on

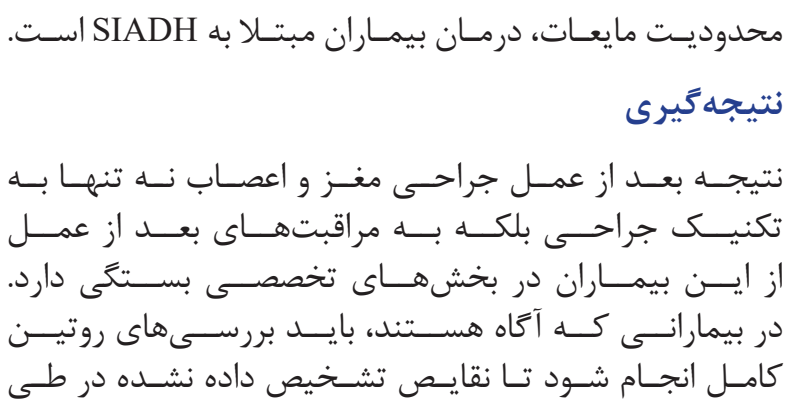

منابع cerebral hemodynamics: its influences on intracranial pressure, cerebral perfusion pressure, and cerebral oxygenation. Neurosurgery. 2004 Mar 1; 54(3): 593-8.

12. Teoh WH, Goh KY, Chan C. The role of early tracheostomy in critically ill neurosurgical patients. Annals-Academy of Medicine Singapore. 2001 May 1; 30(3): 234-8.

13. Kim MK, Baek CW, Kang H, Choi GJ, Park YH, Yang SY, Shin HY, Jung YH, Woo YC. Comparison of emergence after deep extubation using desflurane or desflurane with remifentanil in patients undergoing general anesthesia: a randomized trial. Journal of clinical anesthesia. 2016 Feb 1; 28: 19-25.

14. Odefey AS, Moore LE. The Intrahospital Transport of Neurosurgical Patients. InEssentials of Neurosurgical Anesthesia \& Critical Care 2020 (pp. 537-541). Springer, Cham.

15. Cook TM, Woodall N, Frerk C, Fourth National Audit Project. Major complications of airway management in the UK: results of the Fourth National Audit Project of the Royal College of Anaesthetists and the Difficult Airway Society. British journal of anaesthesia. 2011 May 1; 106(5): 617-31.

16. Lonjaret L, Guyonnet M, Berard E, Vironneau M, Peres F, Sacrista S, Ferrier A, Ramonda V, Vuillaume C, Roux FE, Fourcade O. Postoperative complications after craniotomy for brain tumor surgery. Anaesthesia Critical Care \& Pain Medicine. 2017 Aug 1; 36(4): 213-8.

17. Caceres JA, Goldstein JN. Intracranial hemorrhage. Emergency medicine clinics of North America. 2012 Aug; 30(3): 771.

18. Jiang Y, Ye ZP, You C, Hu X, Liu Y, Li H, Lin S, Li JP. Systematic review of decreased intracranial pressure with optimal head elevation in postcraniotomy patients: a meta-analysis. Journal of advanced nursing. 2015 Oct; 71(10): 2237-46.

19. Manno EM. Airway and pulmonary management in neurosurgical critical care. InEssentials of Neurosurgical Anesthesia \& 
Critical Care 2020 (pp. 565-568). Springer, Cham.

20. Sharrock MF, Rosenblatt K. Acute Airway Management and Ventilation in the Neurocritical Care Unit. InNeurointensive Care Unit 2020 (pp. 31-47). Humana, Cham.

21. Li J, Wu X, Liu H, Huang Y, Liu Y. The effects of protective lung ventilation on regional cerebral oxygen saturation in intracranial tumor operation during dura opening: study protocol for a randomized controlled trial. Trials. 2020 Dec; 21(1): 1-8.

22. Saberi B. Hypertension Induction and Avoidance in the Patients with Neurosurgical Pathologies and Concomitant Cardiovascular Disorders. Arch Neurol \& Neurosci. 2019; 4(2).

23. Kosnik EJ, Hunt WE. Postoperative hypertension in the management of patients with intracranial arterial aneurysms. Journal of neurosurgery. 1976 Aug 1; 45(2): 148-54.

24. Steiner LA, Czosnyka M, Piechnik SK, Smielewski P, Chatfield D. Continuous monitoring of cerebrovascular pressure reactivity allows determination of optimal cerebral perfusion pressure in patients with traumatic brain injury. Critical care medicine. 2002 Apr 1; 30(4): 733-8.

25. Silverstein JW, Rosenthal A, Kwan K, Wagner K, Ellis JA. Application of Multimodal Neuromonitoring in Posterior InferiorCerebellarArtery AneurysmClippings: Review of Two Cases. Cureus. 2020 Mar 17; 12(3).

26. Burkhart CS, Steiner LA. Altered Mental Status in Neurosurgical Critical Care. InEssentials of Neurosurgical Anesthesia \& Critical Care 2020 (pp. 545-550). Springer, Cham.

27. Wagner A, Schebesch KM, Isenmann S, Steinbrecher A, Kapapa T, Baldaranov D, Grauer O. Interdisciplinary decision making in hemorrhagic stroke based on CT imaging-Differences between neurologists and neurosurgeons regarding estimation of patients' symptoms, GCS and NIHSS. Frontiers in Neurology. 2019; 10: 997.

28. Gottschalk A, Berkow LC, Stevens RD, Mirski M, Thompson RE, White ED. Prospective evaluation of pain and analgesic use following major elective intracranial surgery. Journal of neurosurgery. 2007 Feb 1; 106(2): 210-6.

29. Levo H, Pyykkö I, Blomstedt G. Postoperative headache after surgery for vestibular schwannoma. Annals of Otology, Rhinology \& Laryngology. 2000 Sep; 109(9): 853-8.
30. Spektor S, Fraifeld S, Margolin E, Saseedharan S, Eimerl D, Umansky F. Comparison of outcomes following complex posterior fossa surgery performed in the sitting versus lateral position. Journal of Clinical Neuroscience. 2015 Apr 1; 22(4): 705-12.

31. Sudheer PS, Logan SW, Terblanche C, Ateleanu B, Hall JE. Comparison of the analgesic efficacy and respiratory effects of morphine, tramadol and codeine after craniotomy. Anaesthesia. 2007 Jun; 62(6): 555-60.

32.HassaniE, MahooriA, SaneS, TolumehrA.Comparison the effects of paracetamol with sufentanil infusion on postoperative pain control after craniotomy in patients with brain tumor. Advanced biomedical research. 2015; 4.

33. K. P. Kelly, M. C. Janssens, J. Ross, E. H. Horn, Controversy of non-steroidal antiinflammatory drugs and intracranial surgery: et ne nos inducas in tentationem: British Journal of Anaesthesia, 2011September, 107(3) :302-05

34. Can BO, Bilgin H. Effects of scalp block with bupivacaine versus levobupivacaine on haemodynamic response to head pinning and comparative efficacies in postoperative analgesia: A randomized controlled trial. Journal of International Medical Research. 2017 Apr; 45(2): 439-50.

35. Anderson DE, Duletzke NT, Pedigo EB, Halsey MF. Multimodal pain control in adolescent posterior spinal fusion patients: a double-blind, randomized controlled trial to validate the effect of gabapentin on postoperative pain control, opioid use, and patient satisfaction. Spine deformity. 2020 Feb 5: 1-9.

36. Gupta RK, Makkar R, Lamba PS. comparison of effectiveness of intravenous palonosetron versus ondansetron in prevention of postoperative nausea and vomiting in laparoscopic surgeries under general anaesthesia: a randomised double blind interventional study. International Journal of Scientific Research. 2020 Jan 29; 9(1).

37. Kranke P, Wilhelm W, Eberhart L. Management of Postoperative Nausea and Vomiting (PONV). InEnhanced Recovery After Surgery 2020 (pp. 195-202). Springer, Cham.

38. Zhang M, Parikh B, Dirlikov B, Cage T, Lee M, Singh H. Elevated risk of venous thromboembolism among post-traumatic brain injury patients requiring pharmaceutical immobilization. Journal of Clinical Neuroscience. 2020 Mar

39. Störmann P, Osinloye W, Freiman TM, Seifert V, Marzi I, Lustenberger T. Early Chemical Thromboprophylaxis Does Not Increase the Risk 
of Intracranial Hematoma Progression in Patients with Isolated Severe Traumatic Brain Injury. World journal of surgery. 2019 Nov 1; 43(11): 2804-11.

40. Mindermann T. Empirically adapted or personalized antibiotic prophylaxis in select cranial neurosurgery?. Acta Neurochirurgica. 2020 Sep 2: 1-3.

41. Daniel R, Villuri S, Furlong K. Management of hyperglycemia in the neurosurgery patient. Hospital Practice. 2017 Aug 8; 45(4): 150-7.

42. Bilotta F, Rosa G. Glucose management in the neurosurgical patient: are we yet any closer? Current Opinion in Anesthesiology. 2010 Oct 1; 23(5): 539-43.

43. Pellerin L. Lactate as a pivotal element in neuron-glia metabolic cooperation. Neurochemistry international. 2003 Sep 1; 43(4-5): 331-8.

44. Ilie MD, Raverot G. Craniopharyngioma: Endocrinological Aspects After Surgery. InAdult $\quad$ Craniopharyngiomas 2020 (pp. 145-156). Springer, Cham.

45. Delsoglio M, Pichard C, Singer P. How to choose the best route of feeding during critical illness? Clinical Nutrition ESPEN. 2020 Apr 10.

46. Kalisvaart AC, Prokop BJ, Colbourne F. Hypothermia: Impact on plasticity following brain injury. Brain Circulation. 2019 Oct; 5(4): 169.

47. Heino I, Frantzén J, Rinne J, Girard R, Cao Y, Sajanti A, Katila AJ, Posti JP, Takala RS, Tenovuo O, Koskimäki J. Risk Factors for Recurrent Hematoma After Surgery for Acute Traumatic Subdural Hematoma.
World neurosurgery. 2019 Apr 1; 124: e563-71.

48. Jha RM, Kochanek PM, Simard JM. Pathophysiology and treatment of cerebral edema in traumatic brain injury. Neuropharmacology. 2019 Feb 1; 145: 230-46.

49. Algra AM, Lindgren A, Vergouwen MD, Greving JP, van der Schaaf IC. Procedural clinical complications, case-fatality risks, and risk factors in endovascular and neurosurgical treatment of unruptured intracranial aneurysms: a systematic review and meta-analysis. JAMA neurology. 2019 Mar 1; 76(3): 282-93.

50.KinaciA, MoayeriN, vanderZwanA, vanDoormaalTP. Effectiveness of Sealants in Prevention of Cerebrospinal Fluid Leakage after Spine Surgery: A Systematic Review. World Neurosurgery. 2019 Jul 1; 127: 567-75.

51. Venkat P, Chopp M, Chen J. Blood--brain barrier disruption, vascular impairment, and ischemia/ reperfusion damage in diabetic stroke. Journal of the American Heart Association. 2017 Nov 6; 6(6): e005819.

52. Rossi S, Picetti E, Zoerle T, Carbonara M, Zanier ER, Stocchetti N. Fluid management in acute brain injury. Current neurology and neuroscience reports. 2018 Nov 1; 18(11): 74.

53. Niazi T, Browd SR. Surgical Considerations of the Pituitary. InEndocrine Surgery in Children 2018 (pp. 439-448). Springer, Berlin, Heidelberg.

54. Furay EJ, Daley MJ, Satarasinghe P, Lara S, Aydelotte JD, Teixeira PG. Desmopressin is a transfusion sparing option to reverse platelet dysfunction in patients with severe traumatic brain injury. Journal of Trauma and Acute Care Surgery. 2020 Jan 1; 88(1): 80-6. 